\title{
Bringing the IRB into the community: a new framework for the ethical regulation of CBPR
}

\begin{abstract}
The conduct of institutional review boards (IRB) in ensuring ethical protocol is followed has been debated and evaluated for a number of years. The IRB decisionmaking process utilizes a number of regulations in order to protect human subjects in research purposes. Many critics of the system argue that the strict guidelines make it increasingly difficult to retrieve beneficial results and conduct the experiment as initially planned. However, others believe that the standardization involved with the IRB processes makes research practice safer, more transparent, and more effective. Our paper critiques the ethical component of IRB research and its effects on inhibiting or promoting public health practice. In particular, we will address whether current IRB guidelines constrain community-based participatory research.
\end{abstract}

Keywords: IRB, framework, ethical regulation, CBPR, vulnerable populations, prestige custom, flattening, prejudice, issue of scientific merit, life-threatening, permanently disabling, requires inpatient hospitalization, congenital anomaly, cancer, overdose, community predators
Volume 5 Issue 5 - 2017

\author{
Yana Puckett \\ Department of Pediatric Surgery, Saint Louis University and \\ Cardinal Glennon Children's Medical Center, USA
}

\begin{abstract}
Correspondence: Yana Puckett, Department of Pediatric Surgery, Saint Louis University and Cardinal Glennon Children's Medical Center, USA, Tel 7067210427, Email pucketty@slu.edu
\end{abstract}

Received: April 07, 2017 | Published: May 04, 2017
Abbreviations: IRB, institutional review boards; CBPR, community-based participatory research; RERB, research ethics review boards

\section{Introduction}

\section{IRB influence on research}

An Institutional Review Board (IRB) is an appropriately constituted group that has been formally designated to review and monitor biomedical research involving human subjects. ${ }^{1}$ An IRB has the authority to approve, require modification, or disprove research. This group review serves an important role in the protection of the rights and welfare of human research subjects. The purpose of an IRB is to ensure appropriate steps are taken to protect the rights and welfare of humans participating as subjects in research. IRBs use a group process to review research protocols and related materials to ensure the protection of the human subjects. Community-Based Participatory Research (CBPR) is defined as a collaborative approach to research. ${ }^{2}$ CBPR equitably involves all partners in the research process and recognizes the unique strengths that each brings. CBPR begins with a research topic of importance to the community with the aim of combining knowledge and action for social change to improve community health and eliminate health disparities. CBPR proposes assumptions that genuine partnership means co-learning, research efforts include capacity building, findings and knowledge should benefit all partners and CBPR involves long-term commitments to effectively reduce disparities. More pointedly, CBPR has been framed as an orientation to research that focuses on relationships between partners and goals of societal transformation. This research framework is critical in producing impactful studies that can have a major effect on society, yet it is heavily regulated by Institutional Review Boards and their stringent regulation processes.

London explains the controversies which occur in the ethical review process of Institutional Review Boards. Typically, ethical review has excluded vulnerable populations from processes that would determine what type of review process is needed. The new public health is focusing on empowering communities to become the pioneers and managers of their own health. With this evolutionary research, its application and the process of ethical review/decision making must become more inclusive. The responsibility of the IRB is to balance legalist and ethical reasoning methods to safeguard the rights and welfare of participants; and this can be difficult due to cultural differences.

The questions now being asked: Are these ethics committees equipped to address political and cultural differences or are IRBs better suited to address research design? This is both a framework and training question. Are these Review Boards shifting out of a traditional biomedical framework and becoming a learning organization that embodies cultural competency? IRBs are being pushed now to develop new ways to recognize and strengthen the agency of individuals, groups and communities, whom institutional review has classified as vulnerable. However, because of issues involving partiality and power, most decisions of IRBs can be influenced by multiple stakeholders. "Money, power (political or economic), prestige custom, indifference or simple lack of awareness may all influence the decision making process of ethical review. Financial incentives to research and whole institutions may be sufficiently powerful to shape entire research agendas in the developing world and the developed world". ${ }^{3}$ Dubious amounts of research happen only through the support of a grant. Stakeholders and their purses a large percentage of the time, directs IRB decision making. If the stakeholder does not support a certain type of research then it is likely that research will not be pursued.

London also argues what enables differences in power to persist beyond the dollar bill is the lack of explicit recognition of these diverse and powerful interests by the review process. Beyond external influences a "flattening" process needs to happen within IRBs that reduces subjectivities, prejudice, and misunderstandings in the review process. Understanding the pressures and inherit infrastructure of IRBs can help the diffusion of creating opportunity in involving communities to increase the protection of vulnerable populations. 
To add another layer of complexity, the 'vulnerable' require special justification to participate in human subject research in order to avoid human rights abuse. It is IRBs position to ensure that selection of subjects is equitable but also that there is a cognizant of the special problems of research involving vulnerable populations. Also, the concept of vulnerability and consequently, the criteria designating vulnerable populations remain vague. In the Ruof article, it raises the difficulty in defining vulnerability and the discourse surrounding its utility as a qualifying factor in the allocation of health resources; and its appropriateness as a guiding principle in bioethics.

Some examples of this discourse go back to the issue of labeling. Labeling individuals and or communities as 'vulnerable' risks increase of stigma. But other authors acknowledge in order to capture significant aspects and or experiences of marginalized groups then the term 'vulnerable' must be used. For policymakers, the term 'vulnerable' can point to poor health and a population's diminished sense of dignity suffered by the results of unjust public policies and practices. It is a Catch 22 for an organization, let alone an IRB, to use the term 'vulnerable' because of the diverse interpretations.

Within the realm of medical research, there are some who believe vulnerable subjects are cognitively impaired or subject to intimidation. Historically, the devalued vulnerable have served as unwitting subjects in research benefiting 'privileged members of society'. ${ }^{4}$ Here, this not only proposes stigma but by having this framework, it does not capture vulnerability in its entirety. "According to 45 CFR 46, Vulnerable Research Subject Categories include children; prisoners; pregnant women; and handicapped; mentally disabled, economically advantaged or educationally disadvantaged persons". ${ }^{4}$ This gives a broader and more in depth view of what vulnerable is for individuals and populations.

What Ruof also argues is that due to the issue of labeling this has impacted the involvement of IRBs in certain research. Due to fear of being too intrusive, some IRBs have become paralyzed in addressing and or supporting research that involves work which directly impacts vulnerable populations. If this is not addressed, IRBs run the risk of continuing practices that marginalize disparate groups. The Abbot et al. article explains how integral IRBs are to the U.S. system of protection of human research participants. Within the article is a systematic review of empirical studies of U.S. IRBs to determine what is known about the function of IRBs and to identify the gaps of knowledge. It also explains how the IRB process should undergo periodic evaluation. Evaluations can help an IRB determine whether it is effectively protecting human subjects, if it's operating efficiently and if it has adequate authority. The function of an IRB is to review research proposals to assure they adhere to federal regulations, include adequate protections of study participants' right and welfare, and are ethically sound. But little is known about how well IRBs accomplish these goals. The authors do not raise an argument in this study however it raises the question of how effective would it be for diffusion of a new framework which focuses on research that protects populations versus individuals?

Now connecting this question to the Burris et al. article; this article reports the results of qualitative research on how IRBs review their own regulatory processes. In this study, a number of interviews were conducted to assess what IRB members felt could be a means of having a more productive debate about human subject protection. These interviews revealed dissatisfaction from certain board members of IRBs involving the traditional regulatory system; and policies/practices followed about informed consent. Some frustration surrounding the current regulatory system stems from the concept "issue of scientific merit". For some non-clinical social scientist researchers, they come against medically-oriented research ethics review boards (RERB) that require information irrelevant to their research.

Included with this, some respondents from the interviews viewed their RERB's policies and practices about informed consent as 'broken' and in need of major reform. Some respondents mentioned the concern of length of the forms as being a discouragement to potential participants of the research. For example, a social science researcher observed that low-income, non-English speaking families were turned off by the consent forms because they could not read them and they were too long. With these examples we can see what IRBs are faced with. Compounding the issues of understanding how to operationalize protection of vulnerable populations; evolve from a clinically oriented framework to a culturally sensitive framework; and shift from an individual to population mindset, there is a natural resistance to community based research.

Wallerstein et al. ${ }^{2}$ presents community-based participatory research as an alternative research paradigm, which integrates education and social action to improve health and reduce health disparities. CBPR is an orientation to research that focuses on relationships between academic and community partners, with principles of colearning, mutual benefit, and long-term commitment and incorporates community theories, participation, and practices into research efforts. CBPR proposes a set of principles that promotes the concept of shared governance in the decision making, practice and assurance of community based intervention. As CBPR matures, tensions have become recognized that challenge the mutuality of the research relationship, including issues of power, privilege, participation, community, community consent, racial and or ethnic discrimination, and the role of research in social change. This article also addresses the need for cultural humility in order for CBPR to work. Cultural humility refers to "a lifelong commitment to self-evaluation and selfcritique" to redress power imbalances and "develop and maintain mutually respectful and dynamic partnerships with communities. ${ }^{6}$ In all, the critique being made by the author is that cultural humility is a necessary component for any institution of power, including IRBs. IRBs are presented with both the opportunity and challenge in finding a way to shift from a traditional ideology of how research should be conducted, to one that does not constrain community-based participatory research.

\section{How the IRB enables ethical research}

Throughout history, unethical research such as studies during the holocaust has been conducted. Following the Holocaust came the Nuremburg code; which set an ethical standard for conducting research on human subjects that the US lay population did not find fitting as doctors should already be following the Hippocratic Oath. ${ }^{7}$ After a wave of unethical research in the United States, the US government, as well as the people saw a need for the protection of human research participants. By the 1970 's, the IRB was created. ${ }^{8}$ The mission of the IRB is to standardize processes which will protect human subjects. The IRB looks at many aspects of research including the comparison of risk to the patient to the benefit of society, ensuring the patient gives consent to participate and that the study has ongoing oversight. ${ }^{8}$ This section will give an overview of how the IRB standardizes a process to enable ethical research. 
The makeup of the IRB can vary across institutions, but there are federal policies in place to ensure the IRB is multidisciplinary. ${ }^{8}$ The IRB must consist of a chairperson, a scientific member, a nonscientific member, a layperson not affiliated with the institution, and a practitioner. ${ }^{8}$ Board members chosen to represent the institution's IRB must be proficient and have ample experience on the aspect they represent. Not only is the IRB horizontally integrated within disciplines, but the board members are also diverse in their race, ethnicity, gender and they are people who understand the needs of their local community. ${ }^{8}$ The number of board members and backgrounds of fields represented can differ across institutions to reflect the size and specialty of the institution itself, but minimally, the IRB must have the five positions listed above.

The IRB protects participants, researchers and the research institutions. ${ }^{9}$ Participants of IRB approved research have reduced physical risks as well as improved confidentiality. This is due to an increase of adherence in ethical aspects such as respect for persons, beneficence, and justice. ${ }^{9}$ Respect for persons is ensuring the individual understands the implications of the study and that the individual gives consent to join the cohort. ${ }^{9}$ Beneficence is making certain the harm to the individual is reduced while the benefit of the study is maximized. ${ }^{9}$ Creating a just study means spreading risks and benefits equally among the participants. ${ }^{9}$ Another way to protect the participant's safety is following the information exchange and storage rules of the Health Information Portability and Accountability Act when private health information about the patient is collected. ${ }^{9}$ From physical risks to paperwork confidentiality, the IRB provides safety for the participant on all aspects.

The researchers and research institutions that gain approval from the IRB improve their safety from bad press, regulatory action, and ethics of their research. ${ }^{9}$ By following the IRB, researchers and institutions may also save money from potential lawsuits and bad press. Gaining approval from the IRB allows researchers to conduct studies with the peace of mind in knowing the study is being conducted with ethics in the forefront. From the participant to the researcher and the research institution, the IRB is meant to protect all parties involved in research. ${ }^{9}$

Research studies can lead to positive, innovative ways to solve issues, but it can also have lasting, negative outcomes. When a study has unfavorable outcomes, the study may cause more harm to the individual than benefit overall for the community. The IRB steps in to protect the cohort when studies have serious adverse events. Prentice and Gordon define an adverse event as "any experience that suggests a significant hazard, contraindication, side effect, or precaution. With respect to human clinical experience, a serious adverse drug experience includes any experience that is fatal or life-threatening, is permanently disabling, requires inpatient hospitalization, or is a congenital anomaly, cancer, or overdose". ${ }^{10}$ Serious adverse events, when reported timely, could call an end to a dangerous study which would prevent any further harm to the cohort. By reporting serious adverse events to the IRB, the IRB can report it to the necessary department or agency. This process is cost-effective as human trial studies need continuous monitoring, but not every minute side-effect needs to be reported up the chain of command while the serious events need to be reported and acted upon instantaneously. ${ }^{10}$

It is argued that the IRB requires too much paperwork and monitoring. ${ }^{8}$ While aggressive, risky research should be monitored closely, the IRB has made the process simpler for those less complex studies. The federal law allows IRBs to have three types of review which include exempt, expedited, and full review. Exemption from full review allows low risk human studies to occur without IRB review. An example of an exempt review is a taste preference study. Even for institutions that have exemption from full review, the board needs to know about all studies that occur so studies are not happening without their knowledge and approval which could possibly be harmful to the participant. An expedited review could be reviewed by one or only a few members of the IRB. Expedited reviews are likely to be minimally revised studies that have already been reviewed by the entire board or something that is of low risk to the participant. A full IRB review is typically a study with a higher possibility of harm to the cohort such as a drug trial. This study must be reviewed and approved by the whole IRB. Institutions are allowed to have these three types of reviews by federal law, but institutions are not required to have all three options.

As there are different approval methods for studies with different risks to the participants, there are also different processes for those differing trials. ${ }^{9}$ The trials with lower risk have less paperwork than those with more risk. The increase in paperwork is due to the increase in complexity and liability. For those studies with lower risk, the IRB could allow the researchers to have verbal or possibly no consent while those trials with more risk will require written consent. ${ }^{9}$ This flexibility for the lower risk studies has decreased the paperwork and possible oversight for studies that receive exempt or expedited review. While the IRB has been working to make the lengthy and complex nature of protecting the patients more concise, there is still a stigma of researchers dreading the IRB. However, the IRB is ideally comprised of a diverse group of experts whom have a great understanding of the needs of their community. Therefore, this board should have the best interest of the community in mind. For researchers who are in need of ways to improve interactions with their IRB, Oakes provided a list of "Fifteen Tips for Improving Interactions with the Institutional Review Board" which can be found in (Table 1).

Table I Oakes fifteen tips for improving interactions with the institutional review board

Carefully plan the ethical aspects of your study from the very beginning-study the Belmont Report.

Attach to your IRB application a cover letter summarizing your study, with special attention to human subject interactions.

Examine university and Office of Human Research Protections (OHRP) Web sites for examples and specific directions.

If you have questions, telephone and talk with your IRB administrator.

Ask yourself if you would honestly want someone you love to participate in your study.

Work hard to ensure that recruitment materials yield equitable and no coercive results.

Write consent forms at an eighth-grade reading level.

Overestimate risks and underestimate benefits. 
Table Continued..

Educate and debrief subjects on the nature, purpose, and findings of your study.

Establish procedures to delink identifying information from main data sets and sources.

Establish procedures to encrypt any and all identifying information and destroy it as soon as possible.

If you disagree with an IRB decision, read the regulations and then ask for an in-person meeting to discuss things.

Remember that research is not a right but a privilege and IRBs are peer review groups.

Educate your local IRB and then volunteer for it.

Never forget that IRBs did not spontaneously appear to frustrate scientists; they are a direct consequence of many documented violations of very basic ethical principles.

The ethical guidance from the IRB may elongate the research process, but the barriers guarantee research will respect the participant by gaining consent, posing minimal harm and maximizing the benefit for society and that all of the participants will have an equal risk of potential risks and benefits from participating. ${ }^{9}$ While researchers can get discouraged from the strenuous IRB process, researchers should remember Oaks' last tip “....that IRBs did not spontaneously appear to frustrate scientists; they are a direct consequence of many documented violations of very basic ethical principles".

\section{How the IRB restricts research}

A good way to show how the IRB limits CBPR research is through a case report published by Malone and associates. ${ }^{11}$ In this case report, a public health research project on prevention of single cigarette tobacco sales in predominantly African American neighborhoods in San Francisco, California where such sales were prevalent was designed by public health researchers. "Single-cigarette sales were viewed as a problem because single cigarettes were readily available and their reduced unit price made it harder for people to sustain cessation". ${ }^{11}$ Community members also felt that such sales increased minors' access to cigarettes as they are cheaper to buy. According to the community, such sales of single cigarettes were rampant in the neighborhoods and most community members were against them.

With that knowledge, the researchers decided to conduct "a systematic assessment of the proportion of convenience stores in the community that sold single cigarettes in violation of state law". ${ }^{11}$ IRB approvals was obtained. Due to the long time to wait for a sale of a single cigarette, the researchers felt it was impractical to wait around single-cigarette sales. Instead, they wanted to make a singlecigarette purchase attempt and document the result for each store in a prospective fashion.

The IRB refused to approve this study for several reasons. First, they condemned having researchers are the study subjects. Second, the IRB felt it was necessary to obtain informed consent from convenience store owners prior to making a purchase. The researchers in the study felt betrayed by the IRB's rejection. In their view, the IRB chose to protect "community predators" over the health of the community itself. In the IRB's view, their proposal's potential risks included exposing illegal behavior and eliciting feelings of being deceived or fears of entrapment. This appraisal is consistent with traditional biomedical ethics and its focus on individual autonomy, privacy, and agency. This case report is a great illustration of how the current IRB system operates within a biomedical framework and is therefore not compatible for community based participatory research. The biomedical principles of autonomy, privacy, and agency were incompatible with carrying out the research project the researchers had in mind.

In addition, the IRB in their case referred the protocol to a risk management team. This leads us to believe that the IRB not only tries to follow the biomedical framework and is in that way incompatible with CBPR, but that oftentimes, the IRB is protecting itself and the institution from legal risks, but at the cost of research and public health initiatives. "The early IRB referral to the university's risk management department, whence we were referred to the legal department, suggests that the project was regarded in some way as a legal risk and a financial threat to the university". ${ }^{11}$ The IRB decision did protect the institution involved protected the convenience stores whose illegal single cigarette sales could continue. Ultimately, the researchers felt that the decision protected the interests of the tobacco industry.

Another way the IRB is not currently functioning well in the research system is through paradoxical encouragement of deceit. ${ }^{12}$ This not only applies to CBPR research, but to biomedical research, as well. In the current climate of research, most researchers who go through the IRB process and feel as though they are not being treated fairly. ${ }^{12}$ This stems from the fact that the IRB process is extremely rigorous, tedious, and aimed to curtail the range of researchers reach.

As a result, an article by Keith-Spiegel and associates describes how the IRB may be leading researchers to become more deceptive than they would in the first place. This issue is relevant to our topic because it highlights the fact that the IRB may be too stringent in its limitations and thus inhibit public health research by posing too many constraints. This in turn, may cause some to try to shortcut the IRB by being deceitful in constructing IRB protocols for review. This point also alludes to the fact that by being stringent and unnecessarily unfair to CBPR researchers, a tension develops between IRB and the researchers that is just not necessary when all the researchers want to do is develop a research protocol to investigate a noble cause designed to protect communities and people. CBPR researchers spend their time working on bettering communities. Experiencing tension and limitation by the IRB is not conducive to a good working environment, and therefore limits research productivity in general. A paper by Khanlou and associates ${ }^{12}$ investigates this tension. In their opinion, there is an overlap and tensions between ethical review guidelines of IRB and researchers. They also agree that most of these guidelines stem from biomedical frameworks and is incompatible with CBPR. As such, a strong tension is created between CBPR researchers and the IRB that needs to be fixed. Finally, perhaps the most important issue with the current IRB institution and CBPR research is that it fully operates under a biomedical framework. The biomedical framework protects 
the rights of an individual such as autonomy; beneficence, justice, and nonmaleficence are simply incompatible with public health research which targets mainly groups of individuals or communities. In such cases, the protection of an individual is not feasible.

For example, obtaining informed consents on all individuals in a community being studied is nothing short of impossible. In addition, the risks of community based research are much smaller than they are for biomedical research. For example, community based researchers employ data that has already been gathered and de-identified or simple surveys to conduct their research rather than injecting new drug treatments, or testing out a new surgical procedure. Therefore, the risk of community based research is small and often times do not warrant informed consent. A study by Flickers and associates highlights the fact that in their institution, the IRB ethical review forms and guidelines overwhelmingly operate within a biomedical framework that rarely takes into account common community based and behavioral research that often takes place in the public health field. They agree that the IRB thus thwarts public health research by not approving their research. ${ }^{13}$ While the IRB is a great institution that protects individuals from unethical behaviors by researchers, the current IRB system is incompatible with CBPR researchers and may be too stringent for biomedical research, as well.

\section{Alternative constructions to IRBs}

As outlined above, there are a number of issues surrounding IRB treatment of CBPR studies. Although IRBs are ill-equipped to effectively evaluate and accept community-based research, they are aware of the concerns surrounding their protocol. Firstly, there is a lack of communication within the IRB and between board members, researchers, and other stakeholders involved in the research. ${ }^{14}$ When interviewing IRB members, researchers found that the primary concerns were the inability to communicate with coworkers and the burden of excessive paperwork surrounding applications as a major barrier in approving studies. Thus, it became easier to deny community-based studies rather than going through extra loopholes and steps for approval. ${ }^{14}$ The increasing tension between researchers and IRB members and how this may lead to less compliance by researchers to adhere to IRB policies. While most participants in an IRB were satisfied with the board's engagement and understanding of research, others questioned whether they were capable of navigating the newer and less traditional types of research. ${ }^{15}$ The study concluded that tensions existed between board members and existing policies needed to change in order to improve oversight. ${ }^{14}$

The issues surrounding the board members extend beyond communication concerns. In certain cases, IRB members fully understand what community-based research entails. However, they are unwilling to compromise and collaborate effectively with researchers in order to approve such studies because of policy constraints and disinterest in extending themselves beyond their requirements as a board. One study attempted to evaluate IRBs' position on this concern. The author contacted 60 IRBS and received a $55 \%$ response rate.$^{15} \mathrm{He}$ determined that many IRBs were attempting to mend relationships between board members and researchers, yet the lack of access to the IRBs lack of transparency is what caused the largest rifts. The author concluded that an open door policy and amiable communication techniques administered by the IRB could mend the relationship and allow researchers to be more inclined to adhere to guidelines..$^{15}$ Many IRBs across the country and the world are attempting to remedy alternative forms of approaching CBPR research through self-evaluation and changes in protocol. Two such examples are found within the Canadian IRB system and Johns Hopkins University's methods.

Historically, Canadian IRBs and Research Ethics Boards have done a better job of comprehensively evaluating CBPR methods and protocol necessary in order to successfully execute CBPR studies. ${ }^{16}$ More importantly, they have been more open to evaluating their existing IRBs with respect to community-based research and make appropriate changes. ${ }^{16}$ After evaluating seven different institutional review boards across Canada, most agree that effective communication and understanding from IRBs on how CBPRs operate is critical to their success. They recognized that, although they had a basic understanding of community-based research, it was difficult with the ingrained IRB framework to approve such studies. ${ }^{16}$ Furthermore, board members were often not fully aware of the negative effects some biomedical studies could have on the community, such as cultural concerns, lack of resources, etc. Thus, these IRB interviewees recommended more transparent communication with researchers in order to approve community-based studies, and they recommended being more versed in CBPR in order to better evaluate biomedical studies and bring the least amount of harm to the community.

Another study, conducted by Johns Hopkins University, strived to better evaluate and integrate CBPR practices into the IRB. It evaluated an existing CBPR study that was done in California by Loma Linda University, and it critiqued their process to determine what methods are best suited for CBPR research and board approval. ${ }^{17}$ Researchers found that, in many cases, IRBs were ill-equipped at reaching out to community-based researchers because of language/cultural barriers, lack of understanding of what types of intervention they wanted to adapt, and lack of resources. Furthermore, the IRB did little to fully understand the effects of using human subjects for an intervention could have on the entire community. ${ }^{17}$ The study highly recommended more language-based training programs for both researchers and IRB board members to more effectively communicate their concerns. It also made recommendations for training individuals to be more culturally competent and comfortable in working with the community while adhering to IRB guidelines. The university was open to these recommendations and acknowledged that something needed to be changed in this type of evaluation and training process to be more prepared when researchers engaged with the community. ${ }^{17-20}$

\section{Recommendations}

Based on these examples, IRBs are still critically limited in their understanding of CBPR studies. After evaluating the alternative approaches to community-based research, three recommendations have been made to improve IRB intervention in CBPR:

A. More effective training in CBPR: As demonstrated in the study at Loma Linda University, training in community-based research is critical for the community as a study subject and the board to help direct the ethics that surround it. By fully training IRB board members on what it means to be community-based, ethical concerns can be decreased and more community-based studies can be approved by IRBs. It will also instigate collaboration between researchers and board members at an earlier stage in the studyapproval process and in-turn build trust between both of these stakeholders. 
B. Each IRB should have one member fluent in CBPR: All IRBs should have at least one member with a CBPR background when evaluating both CPBR and non-CPBR studies. Evidence has shown that a cross-disciplinary IRB is more effective in approving researching studies. With a CBPR advocate on all boards, the risks and benefits a study could have on the community can be better identified. If an IRB is unable to find a member with this designated background, they can participate in a training protocol for at least one member to be somewhat fluent in CPBR.

C. Improve communication: As demonstrated above, there needs to be more effective communication between researchers and board members in order to demonstrate the strengths of a communitybased study and its impact on society. In many cases, board members already understand these strengths and CBPR processes. If researchers reached out to the IRB at an earlier stage of their study and developed a strong relationship with the board, they could alleviate the concerns IRBs may have with a communitybased study. This, in turn, could help the regulation and approval process be less daunting on the researcher.

These recommendations could greatly improve the scope of CBPR and solidify its importance as a critical, necessary form of research. However, it is important to recognize that IRBs are only in the early stages of recognizing their limitations with CBPR and implementing potential solutions to combat them. Thus, it is difficult to predict the potential ethical concerns that IRBs will deal with when the fully integrate a community-based centered approach to research. Such problems can only be addressed as they emerge. The most crucial step right now is to change the system and guide IRBs in approving more CBPR studies. This will help develop healthier, transparent relationships that can benefit both parties and improve CBPR understanding and regulation under the IRB.

\section{Acknowledgements}

None.

\section{Conflict of interest}

Author declares that there is no conflict of interest.

\section{References}

1. Burris, Scott, Kathryn Moss. US health researchers review their ethics review boards: A qualitative study. $J$ Empir Res Hum Res Ethics. 2006;1(2):39-58.

2. Wallerstein, Nina B, Bonnie Duran. Using community-based participatory research to address health disparities. Health Promot Pract. 2006;7(3):312-323.

3. London, Leslie. Ethical oversight of public health research: can rules and IRBs make a difference in developing countries? Am J Public Health. 2002;92(7):1079-1084.
4. Ruof, Mary C. Vulnerability, vulnerable populations, and policy. Kennedy Inst Ethics J. 2004;14(4):411-425.

5. Abbott, Lura, Christine Grady. A systematic review of the empirical literature evaluating IRBs: what we know and what we still need to learn. J Empir Res Hum Res Ethics. 2011;6(1):3-19.

6. Tervalon, Melanie, Jann Murray-Garcia. Cultural Humility Versus Cultural Competence: A Critical Distinction In Defining Physician Training Outcomes In Multicultural Education. J Health Care Poor Underserved. 1998;9(2):117-125.

7. Oakes J. Risks and Wrongs in Social Science Research. Eval Rev. 2002;26(5):443-479.

8. Enfield, Kyle, Jonathon Truwit. The Purpose, Composition, and Function of an Institutional Review Board: Balancing Priorities. Respir Care. 2008;53(10):1330-1336.

9. Wagner RM. Ethical Review of Research Involving Human Subjects: When and Why Is IRB Review Necessary? Muscle \& Nerve. 2003;28(1):27-39.

10. Prentice, Ernest, Bruce Gordon. IRB Review of Adverse Events in Investigational Drug Studies. IRB. 1997;19(6):1-4.

11. Malone RE, Yerger VB, McGruder C, et al. "It's like Tuskegee in reverse": A case study of ethical tensions in institutional review board review of community-based participatory research. Am J Public Health. 2006;96(11):1914-1919.

12. Azar B. Ethics at the cost of research. Monitor on Psychology. 2002;33(2):38-40.

13. Khanlou N, Peter E. Participatory action research: considerations for ethical review. Soc Sci Med. 2005;60(10):2333-2340.

14. Cook AF, Helena H. Protecting Research Subjects: Irbs In A Changing Research Landscape. IRB. 2011;33(2):14-19.

15. Klitzman, Robert. From Anonymity To "Open Doors": IRB Responses To Tensions With Researchers. BMC Res Notes. 2012;5(1):347-357.

16. Guta, Adrian, Nixon S, et al. "Walking Along Beside the Researcher": How Canadian REBs/IRBs are Responding to the Needs of Community-based Participatory Research. J Empir Res Hum Res Ethics. 2012;7(1):15-25.

17. Spencer-Hwang, Soret S, Halstead L, et al. Making Human Subject Protection Training Community Responsive: Experiences Delivering On The Community-Based Participatory Research Promise. Prog Community Health Partnersh. 2014;8(2):215-224.

18. Flicker S, Travers R, Guta A, et al. Ethical dilemmas in communitybased participatory research: Recommendations for institutional review boards. J Urban Health. 2007;84(4):478-493.

19. Hill JL, Chau C, Luebbering CR, et al. Does Availability of Physical Activity and Food Outlets Differ by Race and Income? Findings from an Enumeration Study in a Health Disparate Region. Int J Behav Nutr Phys Act. 2012;9:105.

20. Keith-Spiegel P, Koocher GP. The IRB paradox: could the protectors also encourage deceit? Ethics Behav. 2005;15(4):339-349. 